\title{
US COVID-19 Vaccines Proven to Cause More Harm than Good Based on Pivotal Clinical Trial Data Analyzed Using the Proper Scientific Endpoint, "All Cause Severe Morbidity"
}

\author{
J. Bart Classen, MD*
}

\begin{tabular}{l|l} 
& "Correspondence: \\
$\begin{array}{ll}\text { Classen Immunotherapies, Inc, } 3637 \text { Rockdale Road, Manchester, } \\
\text { MD }\end{array}$ & $\begin{array}{l}\text { J. Bart Classen, MD, Classen Immunotherapies, Inc, 3637 } \\
\text { E-mail: Classen@vaccines.net. }\end{array}$ \\
& $\begin{array}{l}\text { Received: 24 July 2021; Accepted: } 25 \text { August } 2021 \\
\end{array}$
\end{tabular}

Citation: Classen B. US COVID-19 Vaccines Proven to Cause More Harm than Good Based on Pivotal Clinical Trial Data Analyzed Using the Proper Scientific Endpoint, “All Cause Severe Morbidity”. Trends Int Med. 2021; 1(1): 1-6.

\begin{abstract}
Three COVID-19 vaccines in the US have been released for sale by the FDA under Emergency Use Authorization (EUA) based on a clinical trial design employing a surrogate primary endpoint for health, severe infections with COVID-19. This clinical trial design has been proven dangerously misleading. Many fields of medicine, oncology for example, have abandoned the use of disease specific endpoints for the primary endpoint of pivotal clinical trials (cancer deaths for example) and have adopted "all cause mortality or morbidity" as the proper scientific endpoint of a clinical trial. Pivotal clinical trial data from the 3 marketed COVID-19 vaccines was reanalyzed using "all cause severe morbidity", a scientific measure of health, as the primary endpoint. "All cause severe morbidity" in the treatment group and control group was calculated by adding all severe events reported in the clinical trials. Severe events included both severe infections with COVID-19 and all other severe adverse events in the treatment arm and control arm respectively. This analysis gives reduction in severe COVID-19 infections the same weight as adverse events of equivalent severity. Results prove that none of the vaccines provide a health benefit and all pivotal trials show a statically significant increase in "all cause severe morbidity" in the vaccinated group compared to the placebo group. The Moderna immunized group suffered 3,042 more severe events than the control group ( $p=0.00001)$. The Pfizer data was grossly incomplete but data provided showed the vaccination group suffered 90 more severe events than the control group ( $p=0.000014)$, when only including "unsolicited" adverse events. The Janssen immunized group suffered 264 more severe events than the control group $(p=0.00001)$. These findings contrast the manufacturers' inappropriate surrogate endpoints: Janssen claims that their vaccine prevents 6 cases of severe COVD-19 requiring medical attention out of 19,630 immunized; Pfizer claims their vaccine prevents 8 cases of severe COVID-19 out of 21,720 immunized; Moderna claims its vaccine prevents 30 cases of severe COVID-19 out of 15,210 immunized. Based on this data it is all but a certainty that mass COVID-19 immunization is hurting the health of the population in general. Scientific principles dictate that the mass immunization with COVID-19 vaccines must be halted immediately because we face a looming vaccine induced public health catastrophe.
\end{abstract}

Keywords

Clinical trial, Vaccines, COVID-19.

\section{Introduction}

For decades, true scientists have warned that pivotal clinical trial designs for vaccines are dangerously flawed and outdated
[1]. Vaccines have been promoted and widely utilized under the false claim they have been shown to improve health. However, this claim is only a philosophical argument and not science based. In a true scientific fashion to show a health benefit one would need to show fewer overall deaths during an extended period in the vaccinated group compared to a control group. Less stringent 
indicators of a health benefit would include fewer severe events of all kinds, fewer days hospitalized for any reason, lower heath care expenses of all types, fewer missed days from work for any health reason. No pivotal clinical trial for a vaccine preventing an infectious disease has ever demonstrated an improvement in health using these scientific measurements of health as a primary endpoint. Instead, vaccine clinical trials have relied on misleading surrogate endpoints of health such as infection rates with a specific infectious agent. Manufactures and government agents have made the scientifically disproved and dangerous philosophical argument that these surrogate endpoints equate to a health benefit.

True medical scientists, outside the vaccine fields, have embraced the use of true health measurements as the proven proper scientific endpoint of clinical trials. Decades ago, a pharmaceutical manufacturer would only need to show that a chemotherapeutic agent shrank a tumor or reduce cancer deaths to obtain FDA approval. Manufacturers would market their products under the fraudulent philosophical argument that shrinking tumors or reducing cancer deaths equates to improved survival. However, many of the toxic chemotherapeutic agents would destroy vital organs and actually reduce survival while decreasing cancer deaths at the same time. The FDA and comparable agencies around the world switched to "all cause mortality" as the primary endpoint for pivotal cancer drug trails. The gold standard for marketing approval is to show that those receiving a cancer drug actually live longer than those who do not. Typically, new "miracle" anticancer drugs only prolong survival about 2 months but this added time may be spent severely ill suffering from adverse events caused by the chemotherapy. Application of true scientific principles often severely deflates the hype promoting pharmaceutical products.

All previous vaccine trials have suffered not only from lacking a proper primary clinical endpoint put also from insufficient perspective follow up of adverse events. The trials have failed to account for the well-established toxicity data and epidemiology data that vaccines are associated with chronic immune mediated disorders that may not develop for years after immunization. These adverse events, for example type 1 diabetes, are quite common, develop 3 or more years after immunization, and can exceed the reduction in infectious complications induced by the vaccine as was shown with a hemophilus vaccine [1]. Pivotal trials for the recombinant hepatitis $\mathrm{B}$ vaccine prospectively recorded adverse events for about 7 days after immunization and newer vaccines typically prospectively follow patients 6 months for adverse events.

Use of "all cause morbidity or mortality" as the primary endpoint is warranted in vaccine trials for several reasons. First, the recipients are generally healthy (relative to patients with terminal cancer for example) and the risk of severe morbidity from the target infection is low so even rare adverse events can result in an unfavorable risk benefit. Second, stimulating the immune system with a vaccine can lead to almost any type of adverse event including increasing the incidence or severity of diseases already present in the population. One needs a trial design with a primary endpoint that captures both a decline in infectious complications as well as small rises in hundreds of different immune modified disorders of similar or worse severity as the infectious complications.
Three COVID-19 vaccines are approved by the US FDA under Emergency Use Authorization (EUA). These vaccines have been developed by Pfizer-BioNTech, Moderna, and Janssen. Since marketing has begun multiple reports of potential, adverse events have been recorded. These reports include prion disease [2,3] , clotting disorders [4], myocarditis, reproductive issues, death and many more. A clear difference in frequency of adverse events between different COVID-19 vaccines has been published [3]. The clinical trial designs of the pivotal trials and the resulting data was evaluated to determine if scientifically the results support mass immunization with the vaccines for COVID-19. The published data from the manufacturers' own clinical trials was re analyzed using the proper scientific endpoint "all cause severe morbidity".

\section{Method}

Data from all three US COVID-19 vaccines was published in the New England Journal of Medicine [4-6]. Data from these three publications and the accompanying published appendixes provided the bulk of the information analyzed. On rare occasions supplemental data was found on the FDA's website (https://www. fda.gov/advisory-committees/advisory-committee-calendar) in briefing documents pertaining to FDA advisory panel committees for COVID-19 vaccines from Pfizer-BioNTech, Moderna, and Janssen. The scientific primary endpoint, "all severe events", in the treatment group and controls was calculated by adding all severe or life threatening events reported in the clinical trials by the manufacturers. Severe events included both severe cases of COVID-19 and all other severe events in the treatment arm and control arm respectively.

A Chi square analysis using a $2 \times 2$ table was used to calculate statistical $\mathrm{p}$ values. An online statistical chi square calculator (https://www.socscistatistics.com/tests/chisquare) was used. Statistical calculations ignored small differences in total subject number between efficacy and adverse event populations. The randomized number, shown in Table 1, was used as the study population for statistical calculations. In general, the population for adverse events was slightly higher than that for efficacy. Given the statistical significant $p$, values generated (see Table 1), these small differences do not appear to be material.

The FDA document entitled Guidance for Industry Toxicity Grading Scale for Healthy Adult and Adolescent Volunteers Enrolled in Preventive Vaccine Clinical Trials, 2007, provided the following definitions for adverse events.

Grades 3, Severe: Prevents daily activity and requires medical intervention.

Grades 4, Potentially life threatening: ER visit or hospitalization.

\section{Results}

Moderna

The Moderna pivotal Phase III trial results and protocol are published in the New England Journal of Medicine (NEJM) [5]. The primary endpoint was COVID-19 illness starting 14 days after the second dose of vaccine however the trial had a secondary endpoint 
which was patients developing severe COVID-19 symptoms. This later endpoint allowed for a direct comparison to severe adverse events. The study randomized 30,420 individuals, 15,210 were randomized to receive injections with Moderna's mRNA-1273 vaccine and 15,210 were randomized to receive injections with placebo. Two shots were administered 28 days apart. "Solicited" adverse events were collected 7 days after immunization and "unsolicited" adverse events were reported up to 28 days after administration of each vaccine or approximately 56 days after the first dose according to protocol. Because of dropouts, adverse events were recorded on 15,185 vaccinated patients and 15,166 placebo patients (reference 5, appendix table S8). The treatment group had 11 cases of symptomatic COVID-19 infections and 0 cases severe COVID-19 infections (reference 5, appendix table S13). There were 234 cases of severe "unsolicited" adverse events in the treatment group (reference 5, appendix table S8), and an additional 3,751 "solicited" severe or life threatening (Grade 3 or Grade 4) adverse events (reference 5, appendix table S3 and S4). By contrast, the control group had 185 cases of symptomatic COVID-19 infections and 30 cases of severe COVID-19 infections. However, only one of these case of COVID-19 out of 15,166 controls required admission to an intensive care unit (see reference 5, appendix table S13). There were 202 cases of severe "unsolicited" adverse events in the placebo group and an additional 711 "solicited" severe or life threatening (Grade 3 or Grade 4) adverse events. There were 3 deaths in the placebo group and 2 in the vaccinated group (reference 5, appendix table S8).

\section{Pfizer-BioNTech}

The Pfizer-BioNTech (Pfizer) pivotal Phase III trial results are published in the New England Journal of Medicine [6]. The Pfizer trial was classified as a Phase $1 / 2 / 3$ trial. Two shots were administered 21 days apart. The primary endpoint was confirmed COVID-19 infections 7 days after the second dose. A post hoc analysis of severe COVID-19 infections was included in the appendix published by the NEJM. The study randomized 43,548 individuals of which 100 did not receive injections, 21,720 received injections with the vaccine and 21,728 received injections with placebo. "Solicited" adverse events were collected 7 days after immunization and "unsolicited" severe adverse events were reported up to 14 weeks after administration of the second dose. However, median safety follow up for "unsolicited" events was only approximately 2 months after the second dose at the time of publication in the NEJM. In the treatment arm there was 1 case of severe Covid-19 (reference 6, appendix table S5), 240 "unsolicited" severe adverse events and 21 "unsolicited" life threatening adverse events (reference 6, appendix table S3). In the placebo arm, there were 9 cases of severe COVID-19, 139 "unsolicited" severe adverse events and 24 "unsolicited" life threatening adverse events. Pfizer used a safety subset of approximately 8,183 (both vaccinated and unvaccinated) to record "solicited" adverse events at 7 days. These data that are not shown in Table 1 in part because the data was depicted graphically in the NEJM manuscript. However, graphical data in the NEJM strongly

Table 1: All Cause Severe Morbidity

\begin{tabular}{|c|c|c|c|c|c|c|}
\hline & Moderna & & Control & & Difference & P value \\
\hline Randomized & 15,210 & & 15,210 & & & \\
\hline Days of Safety Follow Up & 56 & & 56 & & & \\
\hline \# Severe COVID-19 Cases & 0 & & 30 & & & \\
\hline \# Unsolicited Severe Adverse Events & 234 & & 202 & & & \\
\hline \# Solicited Grade 3 AE, Shot 1 & 848 & & 361 & & & \\
\hline \# Solicited Grade 4 AE, Shot 1 & 5 & & 6 & & & \\
\hline \# Solicited Grade 3 AE, Shot 2 & 2884 & & 341 & & & \\
\hline \# Solicited Grade 4 AE, Shot 2 & 14 & & 3 & & & \\
\hline \# Total Severe Events & 3985 & & 943 & & 3042 & $\mathrm{p}=0.00001$ \\
\hline \multirow[t]{2}{*}{ \#Deaths } & 2 & & 3 & & & \\
\hline & Pfizer & & Control & & Difference & P value \\
\hline Randomized & 21,720 & & 21,728 & & & \\
\hline Days of Safety Follow Up & 81 & & 81 & & & \\
\hline \# Severe COVID-19 Cases & 1 & & 9 & & & \\
\hline \# Unsolicited Severe Adverse Events & 240 & & 139 & & & \\
\hline \# Unsolicited Life Threatening Adverse Events & 21 & & 24 & & & \\
\hline \# Total Severe Events & 262 & & 172 & & 90 & $\mathrm{p}=0.000014$ \\
\hline \multirow[t]{2}{*}{ \#Deaths } & 2 & & 4 & & & \\
\hline & Jansen & Jansen & Control & Control & Difference & P value \\
\hline Randomized & 19,630 & & 19,691 & & & \\
\hline Safety Subset & & 3,356 & & 3,386 & & \\
\hline Days of Safety Follow Up & 28 & & 28 & & & \\
\hline \# Severe COVID-19 Cases & 21 & & 78 & & & \\
\hline \multicolumn{7}{|l|}{ \# Solicited Grade 3 Adverse Events } \\
\hline Local (extrapolated) & 135 & 23 & 35 & 6 & & \\
\hline Systemic (extrapolated) & 357 & 61 & 122 & 21 & & \\
\hline \# Unsolicited Grade 3-4 Adverse Events & 83 & & 96 & & & \\
\hline \# Total Severe Events & 595 & & 331 & & 264 & $\mathrm{p}=0.00001$ \\
\hline \# Deaths & 3 & & 16 & & & \\
\hline
\end{tabular}

Trends Int Med, 2021 
indicates the vaccinated group has more "solicited" adverse events of all grade levels than the control group.

\section{Janssen}

The Janssen pivotal Phase III trial design and trial results are published in the New England Journal of Medicine [4]. The primary endpoint was prevention of molecularly confirmed, moderate to severe-critical COVID-19 14 days post vaccination however a secondary endpoint was prevention of molecularly confirmed, severe-critical COVID-19 14 days post vaccination. This later endpoint allowed for a direct comparison to severe adverse events. The study randomized 19,630 to receive a single injection with Janssen's adenovirus COVID-19 vaccine and randomized 19,691 to receive a single injection with placebo. "Solicited" adverse events were collected 7 days after immunization and "unsolicited" adverse events were reported up to 28 days after administration of the single dose of vaccine. The treatment group had 21 cases of severe or critical COVID-19 infections while the placebo control group had 78 (reference 4, appendix table S9). Further analysis shows that only 2 of 19,514 immunized patients needed medical intervention for COVID-19 infections starting 14 days after immunization, while only 8 of 19,544 controls needed medical intervention for COVID-19 infections starting 14 days after placebo injection where the COVID-19 infection was confirmed by a central lab (reference 4, appendix table S10). There were 83 "unsolicited" and approximately 492 "solicited" serious adverse events in the vaccinated group compared to 96 "unsolicited" and approximately 157 "solicited" serious adverse events in the control group (reference 4 , appendix table S7). There were 3 deaths in the treatment group and 16 in the control group (reference 4, appendix table S7).

Janssen did not collect "solicited" adverse events from the whole group at day 7 but instead collected these adverse events from a safety group comprising 3,356 vaccinated and 3,380 control patients. FDA briefing document Table 23, page 39 [7] provided the number of "solicited" Grade 3 adverse events in each group. These figures as well as the number of patients randomized were used to extrapolate the number of solicited severe adverse events in the full vaccinated and placebo group as recorded in Table 1.

\section{Discussion}

Scientific analysis of the data from pivotal clinical trials for US COVID-19 vaccines indicates the vaccines fail to show any health benefit and in fact, all the vaccines cause a decline in health in the immunized groups. Health is the sum of all medical events or lack there of. COVID-19 vaccines are promoted as improving health while in fact there is no evidence that these vaccines actual improve health in the individual or population as a whole. The current analysis used the proper scientific endpoint of "all cause severe morbidity", a true measure of health. By contrast, manufactures and government officials promote the vaccines using a surrogate measure of health, severe infections with COVID-19, and the disproved philosophical argument that this surrogate endpoint equates to health. This substitution of philosophy for science is extremely dangerous and is certainly leading to a catastrophic public health event.
Review of data from the three COVID-19 vaccines marketed in the US shows complete lack of a health benefit and even an increase in severe events among vaccine recipients. The proper scientific clinical trial endpoint, "all cause severe morbidity" was created by combing all severe and or life threatening events, both infectious and non-infectious, occurring in the vaccinated and placebo control groups respectively. The data (Table 1) shows there are clearly more severe events in the vaccinated groups. The results are highly statistically significant. The use of a true scientific measure of health as an endpoint for a vaccine trial gives a contrasting result compared to the use of a non-scientific surrogate endpoint of heath, severe infections with COVID-19.

Clinical trial data show there were actually few very "severe" cases of COVID-19 in either the vaccinated or the placebo group. Moderna data shows that only one of 15,166 unvaccinated patients required admission to an intensive care unit for COVID-19. Data provided by Janssen shows that only a few of the "severe" COVID-19 infections required medical intervention. Table S10 in the appendix published in the New England Journal of Medicine [4] , shows only 2 of 19,514 patients immunized with the Janssen vaccine needed medical intervention for severe COVID-19 infections starting 14 days after immunization, while only 8 of 19,544 controls needed medical intervention for severe COVID-19 infections starting 14 days after placebo, where the infection was confirmed by a central lab. This benefit, reduction in 6 case of COVID-19 requiring medical intervention, in 19,630 vaccinated patients is simply statistically insignificant in a population that has a hundred fold more severe events of any cause. The Janssen vaccinated group had 595 severe Grade 3 or 4 events in the first 28 days post immunization. Science thus does not support a health benefit with COVID-19 vaccines. All arguments for immunization are purely philosophical and based on false, discredited, assumptions.

Reductions in infection rates, hospitalization rates and even death with COVID-19 are poor surrogate markers for health and are not proper primary endpoints for a vaccine clinical trial. As discussed earlier with cancer treatments, a trial endpoint showing reduced cancer deaths is not equivalent to enhanced survival. One could apply enough radiation (or cytotoxic chemotherapy) to cancer patients to kill all their cancer cells and prevent cancer deaths but these cancer patients would die of radiation sickness (or chemotherapy induced organ failure) faster than if they died naturally of cancer. In the same manner, reducing severe COVID-19 infections does not equate to enhanced survival especially when the vaccine can cause clotting, heart disease and many other severe adverse events. Potential vaccine recipients need to know if the vaccine improves their survival in order for them to make an informed consent to be immunized. Unfortunately, the current studies with COVID-19 vaccines in fact show they cause a decline in health.

The actual health decline caused by the vaccines is probably much worse than what is depicted in Table 1 for many reasons. First manufactures took a haphazardly approach to recording adverse events in contrast to recording a reduction in COVID-19 events. At 
the time of publication, patients were only followed prospectively for approximately 7 days after immunization for "solicited" adverse events, and then relied on "unsolicited" reports of adverse events for approximately 30-60 days after immunization. Serious noninfectious events occurring after this 30-60 day period were not part of the published data. By contrast, infections with COVID-19 were followed indefinitely since the time of immunization. Both Janssen and Pfizer were specifically lax recording adverse events and only recorded "solicited" adverse events at day 7 in a safety cohort representing less than $20 \%$ of the study population. Given that some of the vaccine clinical trials recruited patients in the third world, patients with low education, and potentially even elderly with dementia the patients can not be expected to understand when they may be having an serious event that needs reporting or how to report it. For these and others reason only $5 \%$ of adverse events are generally ever reported [8].

COVID-19 vaccines were released for marketing under a EUA. Use of such a protocol should be reserved for outbreaks of life threatening epidemics. If this were, actually the case with COVID-19 then reduction in "all cause mortality" should be the primary outcome for the vaccine trials and "all cause severe morbidity" should be the secondary endpoint. However, the manufacturers show no evidence of a survival benefit. Deaths in the trials were extremely rare and of 30 deaths, out of roughly 110,000 trial participants, only about 6 deaths were confirmed to have COVID-19 at the time of death. Regrettably, the vaccines did not reduce morbidity but caused an increase in severe events. Worse, the pivotal clinical trials were never designed to show a benefit in "all-cause mortality" or reduction "in all cause severe morbidity". The fact that the trials were never designed to show these health benefits is an admission that those developing the vaccines never expected the vaccines to result in measurable health benefits. Regrettably some manufacturers have published the false claim [6] that the vaccine have been proven to be "effective" and that its now "unethical" to withhold immunization from the control group. They advocate abolishing the control group by immunizing them. This unscientific act only further proves the pharmaceutical industry is unaccountable to any one and does not feel the need to adhere to principles of science, ethics, or public health.

The COVID-19 vaccine pivotal clinical trials were of very short duration and the question exists whether longer-term follow up will reverse the vaccine induced health decline and show a health benefit. The question is purely philosophical. Some manufactures have already threatened to destroy the randomization by immunizing the control group, as stated above, making further scientific study impossible. While it is possible that the vaccines will continue to prevent severe infectious disease long after the immunization, the reality is that immunity wanes with time and vaccine resistant variants keep developing. Another issue is that severe adverse events will continue to occur over time. Given evidence of prion genic activity by both established pathophysiology [2], animal toxicity data [9] and epidemiology data [3] one can expect an increase in adverse events in the vaccinated group for decades.

Yearly booster are unlikely to improve the health outcome with
COVID-19 vaccines. A booster may provide a small incremental benefit in preventing severe COVID-19 infections however, the boosters are likely to cause many more severe adverse events. Looking at the data on secondary injections with the Moderna vaccine (Table 1) there are approximately 3 times as many Grade 3 or 4 adverse events after the second dose than after the first dose. However, this is not the case following the second dose of placebo in the Moderna placebo group. The net is that adding a booster shot is highly unlikely to induce a favorable health benefit that was missing with the first series of immunization.

Government officials are promoting COVID-19 vaccines as a way to stop the epidemic. There is however no scientific data that the COVID-19 vaccines can improve the health of the population. In fact, the data from the clinical trials seems to point in the opposite direction. Given that the population is the sum of the individuals, and the vaccines cause a decline in health in the individuals, then mass immunization is likely to erode the health of the general population, not improve it. Immunization may even cause a selection bias for new variants. Finally, if the COVID-19 outbreak is the result of a bioweapons attack and vaccine resistant variants represent the release of different prototypes then immunization is almost certain to fail [10].

There is an old saying, fool me once shame on you, fool me twice shame on me. This saying can be applied to the COVID-19 mass immunization program. The US anthrax attack of 2001, which originated at US army is Fort Detrick, has demonstrated that there are people in the US government who desire to attack US citizens with bioweapons [10]. According to the chief FBI agent leading the investigation of the US anthrax attack, conspirators were likely not apprehended in part because the investigation was prematurely ended and prior to stopping the investigation, people at the top of the FBI deliberately tried to sabotage the investigation [11]. In the US anthrax attack of 2001, people high in the US government publicly anticipated the anthrax attack as early as 1999 [10]. Similarly with the COVID-19 attack, people high in government anticipated the COVID-19 attack $[12,13]$ several years before the attack took place [10]. There is even data that an effort was made in 2018 to protect certain populations against COVID-19 by immunizing them with MMR vaccine [14].

In such a hostile government environment, the citizens need to individually evaluate the science of immunization with COVID-19 vaccines and not rely on philosophical arguments propagated by government officials. In this case there is no scientific evidence that the COVID-19 vaccines improve the health of the individual, much less of the population as a whole. Mass immunization with COVID-19 vaccines is certainly leading to a catastrophic public health event.

\section{References}

1. Classen JB, Classen DC. Clustering of cases of insulin dependent diabetes (IDDM) occurring three years after 
Hemophilus influenza B (HiB) immunization support causal relationship between immunization and IDDM. Autoimmunity. 2002; 35: 247-253.

2. Classen JB. COVID-19 RNA based vaccines and the risk of prion disease. Microbiol Infect Dis. 2021; 5: 1-3.

3. Classen JB. COVID-19 Vaccine associated Parkinson's disease, a prion disease signal in the UK Yellow Card adverse event database. J Med - Clin Res \& Rev. 2021; 5: 1-6.

4. Sadoff J, Gray G, Vandebosch A, et al. Safety and efficacy of single-dose Ad26.COV2.S vaccine against Covid-19. N Engl J Med. 2021; 384: 2187-2201.

5. Baden LR, El Sahly HM, Essink B, et al. Efficacy and safety of the mRNA-1273 SARS-CoV-2 vaccine. N Engl J Med. 2021; 384: 403-416.

6. Polack FP, Thomas SJ, Kitchin N, et al. Safety and efficacy of the BNT162b2 mRNA Covid-19 vaccine. N Engl J Med. 2020; 383: 2603-2615.

7. FDA Briefing Document, Janssen Ad26.COV2.S vaccine for the prevention of COVID-19. Vaccines and Related Biological Products Advisory Committee Meeting February 26, 2021.
8. Hazell L, Shakir SAW. Under reporting of adverse drug reactions: a systematic review. Drug Saf. 2006; 29: 385-396.

9. Philippens IHCHM, Böszörményi KP, Wubben JA, et al. SARS-CoV-2 causes brain inflammation and induces Lewy body formation in macaques. bioRxiv preprint. 2021.

10. Classen JB. Review of COVID-19 vaccines and the risk of chronic adverse events including neurological degeneration. J Med - Clin Res \& Rev. 2021; 5: 1-7.

11. Richard L. Lambert versus Attorney General Eric Holder, Robert Muller III and others. Eastern District of Tennessee. Case 3:15-cv-00147-PLR-HBG. Filed April 2, 2015.

12. Schoch-Spana M, Brunson E, Chandler $H$, et al. Recommendations on how to manage anticipated communication dilemmas involving medical countermeasures in an emergency. Public health reports. 2018; 133: 366-378.

13. https:/www.usatoday.com/story/news/factcheck/2020/07/29/ fact-check-2017-anthony-fauci-warned-potentialoutbreak/5494601002/

14. Classen JB. COVID-19, MMR vaccine, and bioweapons. Diabetes Complications. 2020; 4: 1-8. 